\title{
Die UNESCO Biosphäre Entlebuch im Bild \\ Präsentation einer Methode zur Analyse visueller Regionalisierungen
}

\author{
Urs Müller, Norman Backhaus, Hans Elsasser, \\ Ulrike Müller-Böker, Zürich
}

\section{Einleitung}

Es ist mittlerweile ein Gemeinplatz, dass visuelle Bilder massgeblich unsere Vorstellungen von «der» Wirklichkeit prägen (REICHE 2003). Erfolgt der Zugang zur Wirklichkeit, wie dies in mediatisierten Gesellschaften der Fall ist, praktisch ausschliesslich über das Bild, verschwindet «die» Wirklichkeit zwangsläufig hinter dem Medium oder im Medium des Bildes. Dennoch werden die Präsentationsweisen bestimmter Sachverhalte von den Bildkonsumierenden oft für die Wirklichkeit gehalten und beeinflussen damit ihr Handeln.

Was für Konsumprodukte im Allgemeinen gilt, trifft auch auf Regionen zu: In der gegenwärtigen «ästhetischen Ökonomie» (BöHME 2001) müssen sie sich kraft ihres Images auszeichnen und abgrenzen. In diesem Artikel werden unterschiedliche Sichtweisen auf die Region Entlebuch vorgeführt, wie sie im Forschungsprojekt «The Power of Images» im Rahmen des Nationalen Forschungsprogramms 48 «Landschaften und Lebensräume der Alpen» und in Partnerschaft mit dem National Centre of Competence in Research (NCCR) North-South erforscht wurden (MülleR 2007). Die Reise durch verschiedene visuelle Welten der Region Entlebuch wird die bekannte Dichotomie zwischen einer romantisierenden Aussen- und einer alltagsnahen Innensicht vor Augen führen (vgl. STREMLow 1998). Es wird aufgezeigt, dass auch in der Region produzierte Publikationen typische Aspekte der Aussensicht aufweisen, nämlich dann, wenn sie auf die (vermuteten) Werte des touristisch interessierten Zielpublikums gerichtet sind. Denn ein wichtiger Aspekt touristischer Aktivitäten stellt ja gerade die Flucht vor den gewöhnlichen, alltäglichen Erfahrungen dar (URRY 2002: 3). Es wird darüber hinaus ein Beispiel betrachtet, welches diese für das touristische Feld orthodoxe visuelle Kommunikation aufbricht und dadurch einen interessanten Weg weist, wie sich eine sich nachhaltig entwickelnde Region ihren Touristen und Touristinnen verständlich machen könnte.

Aus dem humangeographischen Blickwinkel dieses Forschungsprojektes wurden Bildanalysekategorien entwikkelt, welche die Raumwirksamkeit der visuellen Bilder zu erfassen vermögen. Dieses Vorgehen ermöglicht, bildreiche Präsentationen von «Räumen» in ein über- sichtliches Gesamtbild ähnlich einer «Arealstatistik» zu übersetzen und so unterschiedliche, unter Umständen in Konflikt zueinander stehende Vorstellungen hinsichtlich der Aneignung des Raumes herauszuarbeiten.

\section{Bedeutende Räume}

Die Bildung einer Region beruht darauf, dass ein Raumausschnitt aufgrund ausgewählter Erscheinungen (respektive deren Interpretationen) als unterscheidbar von angrenzenden Raumausschnitten beurteilt wird. Regionen sind somit sinnhafte Konstrukte, die durch strukturiertes und strukturierendes Handeln (re-) produziert werden, ein Handeln, das als Regionalisieren bezeichnet werden kann (HARD 1994: 54). Als Momentausdruck von Regionalisierungen sind Regionen im Sinne von Handlungskontexten bzw. -situationen aufzufassen, die verbunden sind mit Bedeutungen, von vagen Vorstellungen bis zu sanktionierten Rechten und Pflichten. Auf der symbolischen Ebene bedeutet Regionalisieren

«Begriffe und Bilder von Regionen herzustellen und diese mit mehr oder weniger Erfolg in die soziale Kommunikation einzufädeln» (HARD 1994: 54).

Touristische Marketingkampagnen oder politischökonomische Standortwerbungen zeugen von solchen Regionalisierungen.

Abstraktionen in Form von Regionen haben für die Handelnden die Funktion, die komplexe konkrete Welt zu überschauen und zu bewältigen. In diesem Sinne sind Regionalisierungen «Weltkomplexitätsreduktionsstrategien» (HARD 1994: 54), bei denen komplexe Gebilde auf möglichst einfache Figuren und Bilder reduziert werden. Jede Wirklichkeit bietet unendlich viele Möglichkeiten der Regionalisierung, die sich aufgrund der unterschiedlichsten Merkmale wie z.B. Sprache, Wohnformen, Art der Landnutzung, Bodentypen, Klima konstruieren lassen. Wie Bedeutungszuweisungen im Allgemeinen bestimmt sich auch die Art der Regionalisierung in erster Linie durch die Interessen der Handelnden. Die «Realität» von Regionen in ihrer Gesamtheit ist letztlich «durch und durch sozial» (BourdiEu 1990: 96). Als Teil der sozialen Strukturen weisen Regionen zwar eine gewisse Persistenz auf, bleiben aber grundsätzlich veränderbar. Werden Regionsbezeichnungen in neuen (visuellen und/oder sprachlichen) Bildern präsentiert, ändern sich auch die Bedeutungen, die diese Begriffe bei den Handelnden haben und damit im Prinzip auch das sie betreffende Handeln. 


\section{Regionalisierungen durch Bilder}

«D'Antlibuecher, die vo hende vöre» (Aussage einer Jugendlichen in: Probst 1999: o.S.) oder das Bild des Entlebuchs als «Armenhaus der Schweiz» (vgl. WICKI \& Kaufmann 2003: 344): Dies sind Stereotype, Klischees. Walter Lippmann beschrieb 1922 Stereotype als «pictures in our head», als

«vereinfachte, relativ rigide und schwer zu verändernde Denkmuster, die mit der äusseren Realität nicht übereinstimmen müssen, jedoch unser Verhalten stärker beeinflussen als die tatsächlichen Bedingungen» (zit. in: GASTGAMPE 1993: 129).

Stereotype sind wichtige Instrumente bei der Reduktion der (Reiz-) Komplexität auf ein «zu bewältigendes Mass» bzw. bei der Kanalisierung der Information in eine «günstige Richtung».

«Die Menschen nehmen (...) Bilder auf und sträuben sich nicht gegen das Denken und Wahrnehmen in Stereotypen. Im Gegenteil - wir sind froh über jede Form der Simplifizierung: Marilyn Monroe, die frivole Blondine, Albert Einstein, der coole Alte. $\mathrm{Zu}$ wenig bemühen wir uns um ein eigenes differenziertes Bild von der Wirklichkeit» (REICHE 2003: 10ff.).

Mit den Stereotypen eng verwandt sind «Images» im Sinne von Produkte-, Personen- oder Regionsimages, d.h. der Gesamtbilder, die sich Individuen von besagtem Objekt (bzw. einem Begriff) machen (vgl. MüLLER $\&$ BACKHAUS 2007).

In unserer visuell dominierten Zeit (vgl. MAAR \& BURDA 2004) prägen und formen Bilder die Wahrnehmungsweisen und zeigen beispielsweise auch, was Regionen bedeuten können. Bilder vermitteln Eindrücke und machen damit Unbekanntes vermeintlich bekannt, wobei immer bestimmte Eigenschaften beleuchtet, andere aber ausgeblendet werden. Die «unbestreitbare Macht der Bilder» (STÄMPFLI 2007: 89) liegt dabei in ihrem Konkretsein begründet. Bilder betrachtet man in der Regel mit der gleichen Leichtigkeit, mit der man sehend durch die Welt schreitet, während das Erfassen und Verstehen von Text eine mehr oder weniger grosse gedankliche Anstrengung verlangt. Fotografien gelten als Garant für eine rein mechanische Nachahmung der Realität, als zweidimensionales, visuelles Double der Realität (vgl. WIEsING 2005). Zwischen Bild und Abbild scheint keine Distanz zu bestehen, jedenfalls keine, die, wie im Falle diskreter digitaler Zeichen (z.B. der Buchstaben und Zahlen), einer Übersetzung bedarf (vgl. ScHOLZ 2004: 126). Bilder wirken deshalb so unmittelbar wie die erblickte Realität. Sie geben dem Betrachter auf schnellstem und leichtestem Wege konkrete und detaillierte Vorstellungen z.B. von Sachen, Ereignissen, Personen. Fotografien sind Zeichen, die den Anspruch erheben, keine Zeichen zu sein, die sich als natürliche Unmittelbarkeit und Gegebenheit maskie- ren (Mitchell 1990:55f.). Auch im Zeitalter verbreiteter Bildmanipulationen nehmen Fotografien noch den Rang eines Beweises ein:

«Etwas, wovon wir gehört haben, woran wir aber zweifeln, scheint <bestätigt>, wenn man uns eine Fotografie davon zeigt» (SonTAG 1978: 11).

\section{Die Methode der Bildinhaltsanalyse}

Die methodische und theoretische Auseinandersetzung mit der Macht der Bilder ist zu einem wichtigen Anliegen der Humangeographie im cultural turn (WERLEN 2003) geworden. Während Einzelbildanalysen in semiotischer, hermeneutischer oder phänomenologischer Tradition bereits verbreitet durchgeführt werden (vgl. u.a. FlitNer 1999; DirksmeIer 2007), fehlen fruchtbare Zugänge zur Analyse bildreicher Publikationen weitgehend bzw. weisen keinen Bezug zu Gesellschafts-Raum-Interaktionen auf (zur Übersicht vgl. Rose 2001: 54ff.). Für die vorliegende Untersuchung wurde eine der Bildinhaltsanalyse angelehnte Methode entwickelt, mit welcher sich raumwirksame Bedeutungszuweisungen in Regionen analysieren und zusammenfassend darstellen lassen. Die Analysekategorien sollen die Bedeutungen, die Menschen dem Raum geben bzw. aus den Bildern lesen (können), integrieren und dadurch die in den Bildern materialisierten Wertstrukturen erfassen. Diese auf die Erfassung der Raumbedeutungen bzw. -funktionen gerichteten Kategorien werden hier als Raumaneignungskategorien bezeichnet.

Die Entwicklung der Raumaneignungskategorien folgt einem Wechselspiel von theoretischen Überlegungen sowie von empirischer Anwendung der Kategorien auf das vorliegende Bildmaterial (die Herleitung der Kategorien ist ausführlich dargestellt in MÜLLER 2007). Da es sich bei der UNESCO Biosphäre Entlebuch (UBE) um eine Modellregion für eine nachhaltige Entwicklung handelt, wurden die Raumaneignungskategorien an die Multidimensionalität der Nachhaltigkeit angelehnt. Damit die kontextspezifischen Bedeutung(en) der Bilder kategorisiert werden konnten, musste ferner ihre verbale Verankerung berücksichtigt werden. Denn mittels Bildlegende, Bildüberschrift oder beistehendem Text wird die Vielfalt möglicher Bedeutungen auf die jeweils beabsichtigten eingeschränkt (BARTHEs 1993: 1421).

Das Resultat der Bildinhaltsanalyse stellt eine bildflächenproportionale Gesamtübersicht über die Art der Raumaneignungen einer ausgewählten Publikation dar (vgl. die folgenden Abbildungen). Wurden beispielsweise sämtliche Bilder als Naturraum kodiert, ergibt sich ein Gesamtbild dieser Region als 100\% «Wildnis». Kommen hingegen Bilder unterschiedlicher 
Kategorien vor, werden diese durch die jeweilige Bildfläche gewichtet zusammengefasst. Die Gesamtschau stellt damit eine verfeinerte und erweiterte «Arealstatistik» der betreffenden Region dar - allerdings nicht auf den «Realraum», sondern auf seine bildliche Präsentation in den analysierten Publikationen bezogen.

\section{Blicke auf die UNESCO Biosphäre Entlebuch (UBE)}

Das Entlebuch wurde am 20. September 2001 von der UNESCO als Biosphärenreservat anerkannt. Ein Jahr zuvor haben sich die Stimmberechtigten aus den acht Gemeinden der Planungsregion Entlebuch positiv zur Anmeldung geäussert (vgl. ScHMID et al. 2004). Biosphärenreservate stellen Versuchsfelder dar, in welchen neue, modellhafte Wege in Richtung einer nachhaltigen Entwicklung erprobt werden (UNESCO 1995: o.S.). Dieser innovative Schritt brachte dem Entlebuch eine noch nie da gewesene Medienpräsenz (vgl. ERNE et al.2003), deren visueller Anteil im Folgenden an ausgewählten Beispielen genauer betrachtet werden soll.

\subsection{Die Sicht der Regionalzeitung «Entlebucher Anzeiger» (EA)}

Medial trat das Biosphärenprojekt am 19. April 1996 in der Regionalzeitung EntLebucher Anzeiger (EA) erstmals in Erscheinung. Der EA erschien während des untersuchten Zeitraums dreimal wöchentlich in einer Auflage von 8'234 Exemplaren (WEMF AG 2003), womit durchschnittlich $80 \%$ der Entlebucher Haushalte erreicht wurden.

In den rund viereinhalb Jahren bis zu den Volksabstimmungen im September 2000 berichtete die Zeitung in 144 Artikeln und einer vierseitigen Zeitungsbeilage über das Vorhaben, die zusammen insgesamt 187 Fotografien und Grafiken aufwiesen. Darin wird ein Entlebuch präsentiert, welches wenig mit klischierten Vorstellungen gemein hat (vgl. Abb. 1): Weder Naturbilder noch Darstellungen einer traditionellen, idyllischen Kulturlandschaft spielen eine bedeutende Rolle. Die Biosphäre Entlebuch wird vielmehr als (moderner) Lebens- und Produktionsraum präsentiert. Der hohe Anteil an Identifikationsraumbildern spiegelt das Erfolgsrezept der Biosphären-Promotoren wider, indem praktisch sämtliche wichtigen Identifikationsfiguren für das Vorhaben gewonnen werden konnten. Weiter erwähnenswert ist die Kategorie «Politraum» (3,7\% der Bildfläche), die den partizipativen und diskursiven Charakter nachhaltiger Entwicklung vermittelt. Auf 12 der 187 Bilder (bzw. 2,2\% der Bildfläche) sind kooperierende Personen bzw. Vertreter kooperierender Institutionen zu sehen (Kategorie «Kooperationsraum»), die aufzeigen, dass sich Kooperationen lohnen können - ein wichtiger Aspekt einer nachhal- tigen Entwicklung. Schliesslich wird auf neun Bildern das Entlebuch als «Problemraum» thematisiert (3\% der Bildfläche). Gezeigt werden unter anderem Konflikte zwischen (Winter-) Sport und Natur- bzw. Moorschutz, die Folgen von Naturgewalten oder das illegale Drainieren von Moorflächen.

Der EA zeigt also ein vielfältiges und grossmehrheitlich kulturräumliches Bild der UBE. Die Präsentation der UBE in der Regionalzeitung EA ist alltagsnah auf die Entlebucher Bevölkerung zugeschnitten. So sind auch die Identifikationsfiguren fast ausschliesslich lokal bis regional bekannte Persönlichkeiten. Viele der «Leitfiguren» stellen sich nicht nur mit ihrem Namen hinter das Vorhaben, sondern weisen konkret einen möglichen Weg, wie die Region vom Biosphärenlabel profitieren kann.

\subsection{Die Sicht der «Schweizer Familie»}

Als zweites Beispiel wird ein Artikel der deutschsprachigen Familienzeitschrift SchweIzer FAMILIE betrachtet, welche in einer Auflage von 165'064 Exemplaren (WEMF AG 2003) einmal wöchentlich erscheint. Sie gehört der Tamedia AG, die u.a. auch den Zürcher TAges-AnZeIgER herausgibt.

Am 13. November 2001, rund drei Wochen nach der Anerkennung der UBE durch die UNESCO, erschien in der SchweIzer FamiLie ein Artikel über das neu entstandene Biosphärenreservat mit dem Titel «In der Natur liegt die Zukunft». Auf den sieben Seiten befinden sich zehn Fotografien und eine Graphik. Die Bilder dieses Artikels stechen wegen ihrer ästhetischen Qualität unmittelbar ins Auge: Die in Szene gesetzten Naturobjekte und -landschaften erhalten durch das Spiel der Farben eine irreale Schönheit. Solchen Bildern, die in der «Tradition des Pittoresken» stehen (HenNig 1999: 106), wird eine starke emotionale Anziehungskraft auf den Bildbetrachter zugeschrieben (vgl. Bucher 2006). Wie Abbildung 2 zeigt, wird im Artikel der Schweizer Familie ein insgesamt sehr naturräumliches Entlebuch gezeigt $(85,8 \%$ der Bildfläche). Die 6,2\% «Harmonieraum» verweisen auf ein ästhetisch inszeniertes Bild einer traditionellen Kulturlandschaft.

Der Artikel aus der Schweizer Familie weist die bekannte Aussensicht auf die Berggebiete auf, welche geprägt ist vom romantisierten und romantisierenden, vom idyllisierten und idealisierenden Blick, der wenig Bezug zum Leben der Menschen in den Gebieten hat. Betrachtet man weitere Zeitungsartikel, die anlässlich der drei bedeutenden Diskursereignisse (Abstimmungsergebnisse, Bekanntgabe der bundesrätlichen Unterstützung der Kandidatur bei der UNESCO und schliesslich die Anerkennung als Biosphärenreservat) in den nationalen Medienerzeugnissen erschienen 


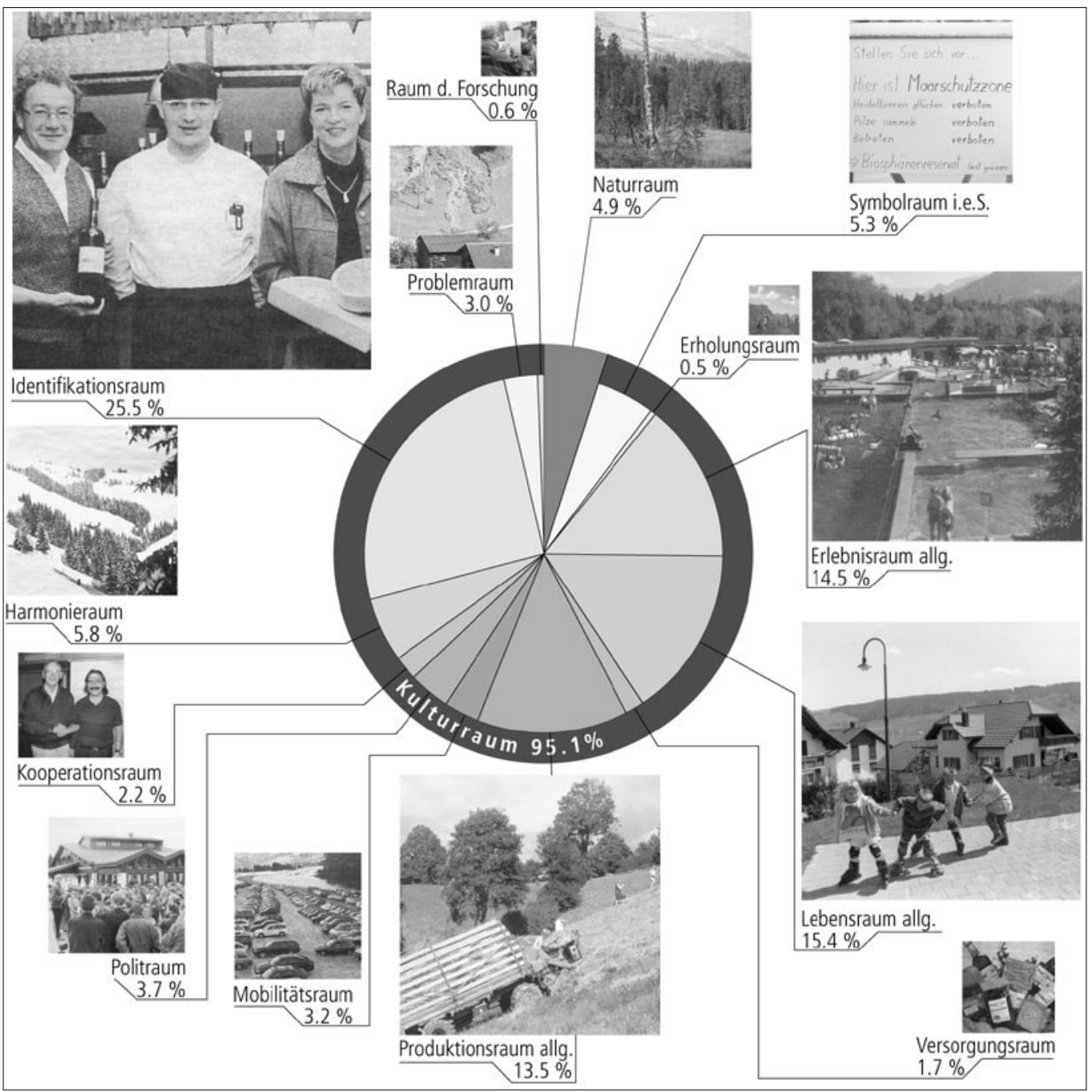

Abb. 1: Das Entlebuch als Lebens- und Wirtschaftsraum. Die UNESCO Biosphäre Entlebuch, wie sie in den Artikeln des EnTLEbucher AnZeigers präsentiert wurde.

Entlebuch as both cultural and economic landscape. The Entlebuch UNESCO Biosphere Reserve as presented in articles published in the local newspaper ENTLEBUCH ANZEIGER.

Entlebuch en tant qu'espace de vie et espace économique. La réserve de biosphère d'Entlebuch (UNESCO) telle qu'elle est présentée dans les articles de l'ENTELBUCH ANZEIGER.

Quelle: EnTLEbucher ANZEIger, 19.4.1997-4.9.2000 (verschiedene Ausgaben); Graphik: U. MüLleR

sind (vgl. ERNE et al. 2003), bestätigt sich das Bild der SchWEIZER FAMILIE: Abgesehen von äusserst wenigen Ausnahmen tradiert die Aussensicht das Bild eines weitgehend natürlichen, idyllischen Entlebuchs. Mit solchen Veranschaulichungen wird der Eindruck vermittelt, dass nachhaltige Entwicklung in einer Modell- region primär Naturschutz bzw. Musealisierung der kulturlandschaftlichen Juwelen umfasst. Die multidimensionale Bedeutung nachhaltiger Entwicklung wird ausgeblendet, die Modellhaftigkeit der regionalen Entwicklungsbestrebungen vereinseitigt. Nachhaltige Entwicklung scheint gleichbedeutend zu sein mit 


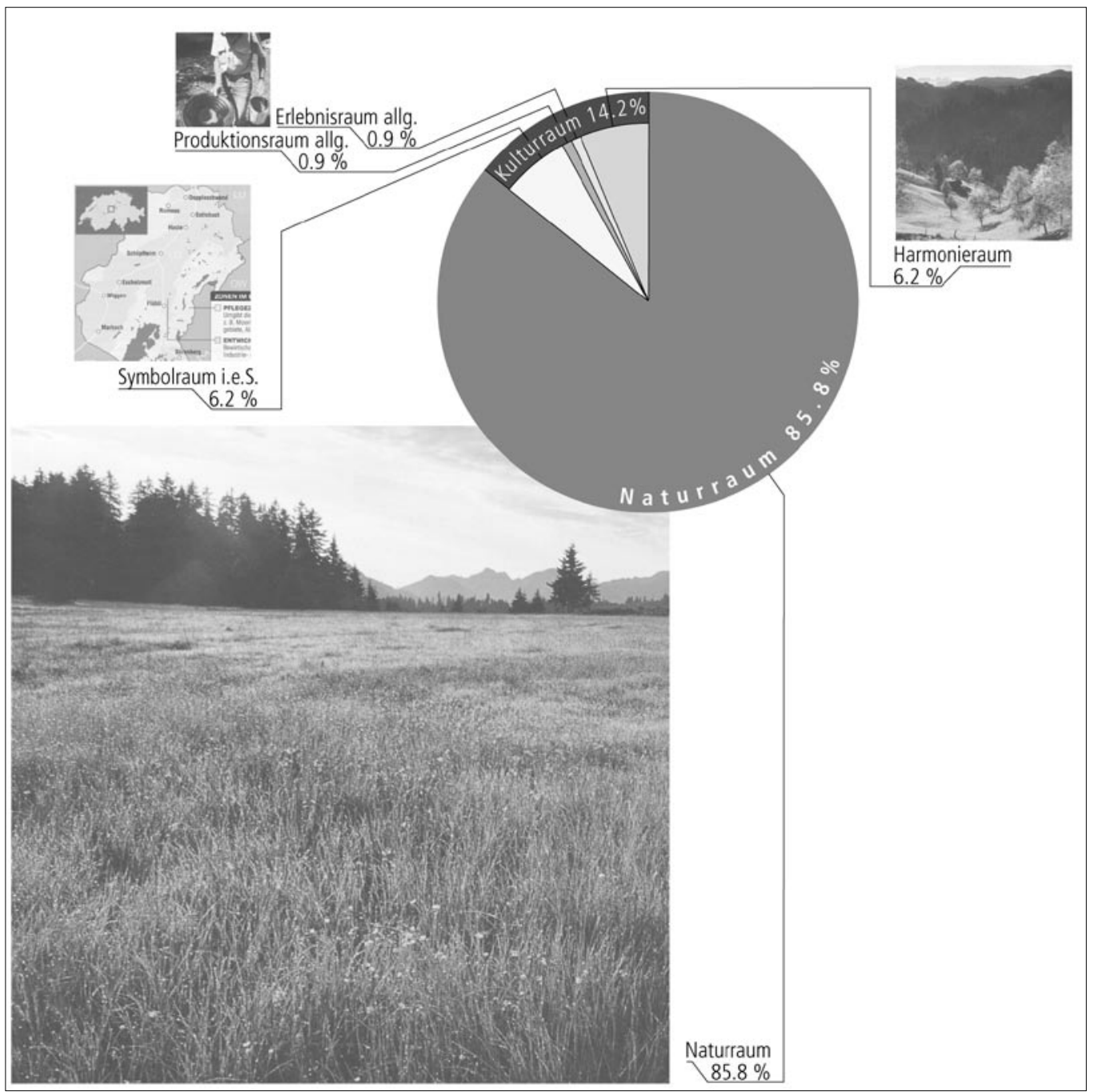

Abb. 2: Das Entlebuch als ein Ort der Natur. Die Präsentation der UNESCO Biosphäre Entlebuch im Artikel «In der Natur liegt die Zukunft» der SChweizer Familie Nr. 37, 2001, S. $22-28$

Entlebuch as ecological landscape. Presentation of the Entlebuch UNESCO Biosphere Reserve in an article titled "Nature is the destiny of the future» in the Swiss magazine SchweIzer FamiLIE No. 37, 2001, p. 22-28

Entelbuch en tant que lieu naturel. La présentation de la réserve de biosphère d'Entlebuch (UNESCO) dans l'article «Le futur est dans la nature» de la SchweIzer FAMILIE, numéro 37, 2000, pp. 22-28

Graphik: U. MÜLLER; Fotos: M. SCHMID

Bewahren und Schützen, ihre progressive und innovative Seite kommt nicht zur Geltung.

\subsection{Die Sicht der «Revue Schweiz»}

Bislang wurde die Diskrepanz zwischen einer den regionalen Informationsinteressen gehorchenden
Innensicht (EA) und einer ästhetisch geprägten Aussensicht, die in der (touristischen) Tradition des romantischen Naturgefallens steht, festgestellt. Als weitere Sicht auf die UBE wird eine dem Entlebuch gewidmete Ausgabe der Revue SchweIz von anfangs 2001 betrachtet. Die Revue SchweIz bezeichnet sich 
als «Das Magazin für Freizeit, Tourismus, Kultur und Natur». Sie berichtet über die aktive Freizeitgestaltung in der Schweiz, über verschiedenste Themen aus allen Regionen der Schweiz, bebildert mit «exklusiven Fotografien von den besten Schweizer Fotografen/-innen» (http://www.revueschweiz.ch/revue_html_seiten/ revueschweiz.html 7.2.2005). Die Revue Schwerz hat eine beglaubigte Gesamtauflage von rund 20'000 Exemplaren (WEMF AG 2003).

In die Auswertung einbezogen wurden die Artikel des redaktionellen Teils der Revue SchweIz, welcher 39 der total 66 Seiten ausmacht (der Rest umfasst deklarierte Werbedarstellungen einzelner Gemeinden der UBE, sonstige Hinweise und Werbung). Auf diesen 39 Seiten kommen 72 Bilder vor (Abb. 3).

Angesichts des touristischen Zielpublikums dieser Publikation dürfte man eine ähnliche Präsentation der UBE wie in der SchweIzer Familie erwarten. So werden denn auch ästhetische Natur- und Landschaftsbilder des Fotografen StePHAN KöllikeR gezeigt, doch alles in allem offenbart sich ein deutlich heterogeneres Bild als im Artikel der SCHWEIZER FAMILIE. Naturraum-Bilder machen in der Revue SchweIz knapp einen Drittel der Bildfläche aus (30,1\%). Sie sind damit weit unter dem Wert der Schweizer FAMILIE. Auch die mitunter touristisch interessanten Kategorien Erlebnis- und Erholungsraum fallen mit zusammen knapp 9\% bescheiden aus. Dagegen nimmt die Bildfläche der Kategorien Lebens- und Produktionsraum allg. zusammen (32,8\%) mehr Bildfläche ein als der Naturraum. Mit Bildern der vier Entlebucher Industriebetriebe zeigt ein bedeutender Anteil der Produktionsraumbilder ein modernes industrielles Entlebuch.

Weshalb präsentiert die Revue SchweIz nicht ein natürlicheres oder touristischeres Entlebuch? Die Ausgaben der Revue Schweiz entstehen in der Regel in Zusammenarbeit mit den porträtierten Regionen, insbesondere mit den Tourismusverantwortlichen, in diesem Fall vom Management der UNESCO Biosphäre Entlebuch. Man habe darauf hingewirkt, dass das Entlebuch in seiner Vielfalt gezeigt werde, wozu das Alltagsleben der Entlebucher und Entlebucherinnen, die Industrie und das Gewerbe gehören. Die UBE, Modellregion für eine nachhaltige Entwicklung, begeht damit eine marketingstrategische Häresie (vgl. Bourdieu 1993: 109): Der für das touristische Feld orthodoxe Weg einer beschönigenden visuellen Kommunikation (beispielhaft im Artikel der ScHWEIZER FAMILIE ersichtlich) wird nicht begangen, sondern die Vielfalt des Entlebuchs erstaunlich offen gezeigt. Schreitet die Modellregion Entlebuch in Richtung einer neuen, unorthodoxen Tourismuswerbung, welche es wagt, die (ländlichen) Zielorte nicht (nur) museali- siert, ästhetisiert, idealisiert zu präsentieren? Gelingt es der UBE, ihre Leistungen im Bereich nachhaltige Entwicklung (z.B. Regionalprodukte, Kooperationen, Bildungsprogramme) umfassend zu präsentieren und sich über diese Differenz auf dem umkämpften Tourismusmarkt zu behaupten? Eine visuelle Kommunikation, welche der Multidimensionalität der nachhaltigen Entwicklung gerecht wird, ist zweifelsfrei ein interessanter Versuch, das Wissen und Handeln der Touristen und Touristinnen in Richtung Nachhaltigkeit zu beeinflussen.

\section{Fazit}

Mit dem Biosphären-Vorhaben wurde die nationale und internationale Medienbühne aufmerksam auf das Entlebuch. Die Art und Weise, wie das Entlebuch von der Aussensicht (Artikel der SchweIzer FAMILIE) präsentiert wird, unterscheidet sich allerdings frappant von der Innensicht. Die von den Verantwortlichen der SchweIzer Familie getroffene Bildwahl bleibt den Klischees eines natürlichen und idyllischen Entlebuchs verhaftet. Solche Präsentationen in Form von Naturräumen oder arkadischen Kulturlandschaftsidyllen sprechen potenzielle Touristen und Touristinnen an, blenden aber die Herausforderungen und Leistungen der Entlebucher Bevölkerung auf dem Weg in Richtung einer nachhaltigen Entwicklung ihrer Region aus. Die Sichten des Entlebucher Anzeigers und der Revue SchweIz dagegen stellen dem Bild rückständiger, träger Agrargesellschaften das Bild dynamischer, eigenverantwortlicher Unternehmer und Unternehmerinnen beiseite.

In diesem Artikel wurde eine Möglichkeit vorgestellt, wie sich bildreiche Publikationen aus einer humangeographischen Perspektive analysieren und interpretieren lassen. Die Bildanalysen eröffnen einen Zugang zu den oft unreflektierten «Bildern in den Köpfen» der Bildproduzierenden und Bildrezipierenden, die aus Sicht der Strukturationstheorie die eigentlichen Handlungsmotivationen darstellen (vgl. GidDENS 1984). Denn die Vorstellungen, die Handelnde von der Welt haben, werden entscheidend durch Medien aller Art geprägt: z.B. durch Fernsehberichte, Filme, Zeitungen, Magazine, Werbung. Angesichts der menschlichen Beschränktheit, Aspekte aus der unendlichen Vielfalt der Welt «mit eigenen Augen zu sehen», scheint die Aussage LuHManns berechtigt:

«Was wir über unsere Gesellschaft, ja über die Welt, in der wir leben, wissen, wissen wir durch die Massenmedien» (Luhmann 1996: 9).

Die Analyse der Medienerzeugnisse ergibt deshalb wichtige Einblicke in die (wahrscheinlichen) Weltbilder der Handelnden. 


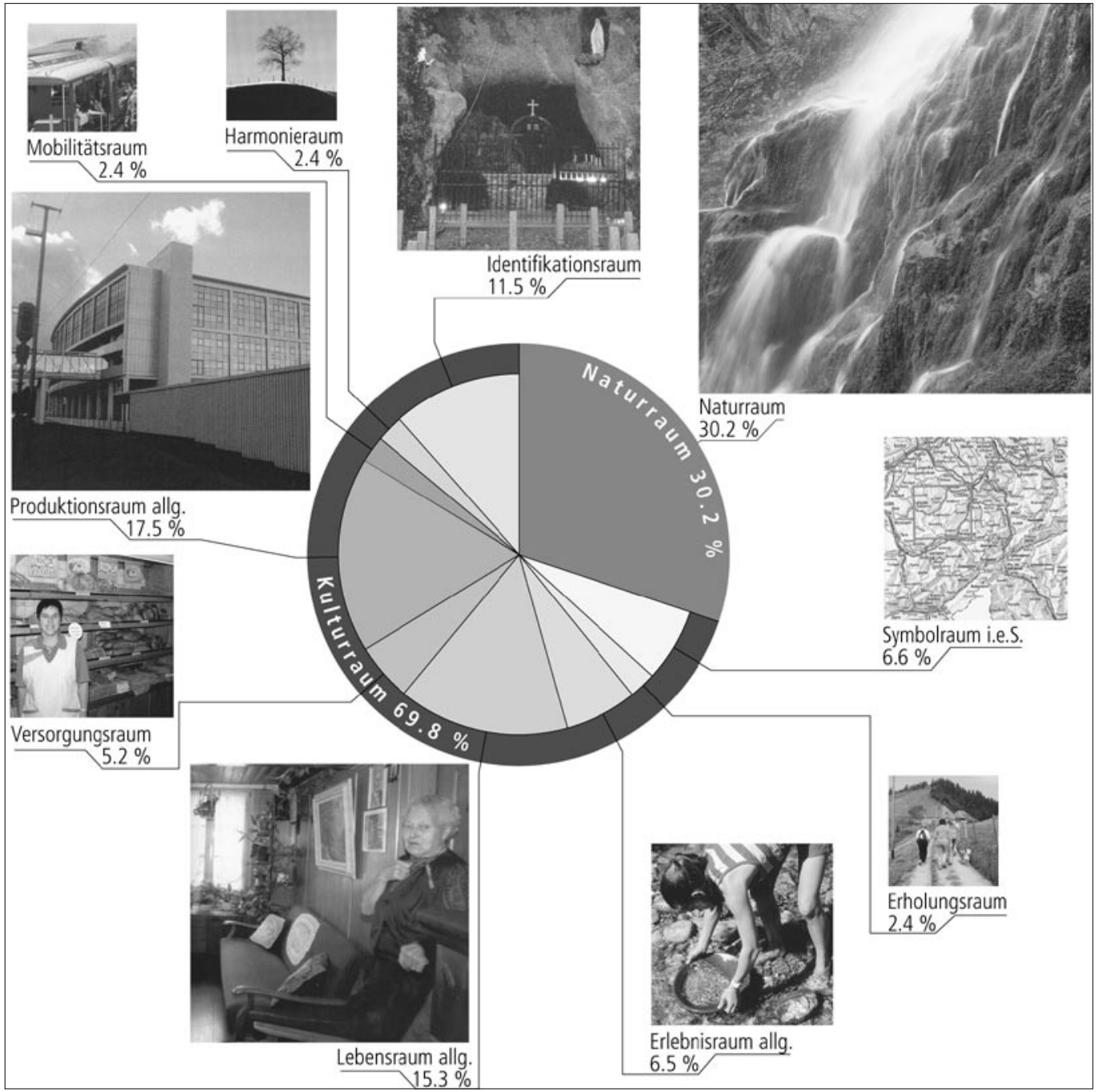

Abb. 3: Unorthodoxe Präsentation der UNESCO Biosphäre Entlebuch im Tourismusmagazin REvUE ScHwEIz Unorthodox presentation of the Entlebuch UNESCO Biosphere Reserve in the Swiss tourist magazine REVUE SCHWEIZ Une présentation non orthodoxe de la réserve de bioshpère d'Entlebuch (UNESCO) dans le magazine de tourisme Revue SchweIz

Graphik: U. Müller; Fotos: Revue SchweIz, Nummer 1/2001

\section{Literatur}

BARTHES, R. (1993/Orig. 1964): Rhétorique de l'image. - In: Marty, E. (Hrsg.): Roland Barthes: œuvre complètes. - Tome 1: 1942-1965, Paris: Editions du Seuil: 1417-1429.

BöHme, G. (2001): Aisthetik: Vorlesungen über Ästhetik als allgemeine Wahrnehmungslehre.-München:W. Fink.
Bourdieu, P. (1990/Orig. 1982): Was heisst sprechen? Die Ökonomie des sprachlichen Tauschs. - Wien: Braumüller.

Bourdieu, P.(1993/Orig. 1976): Über einige Eigenschaften von Feldern. - In: Bourdieu, P. (Hrsg.): Soziologische Fragen. - Frankfurt am Main: Suhrkamp: 107-114. Bucher, O. (2006): Das so genannte Schöne - An- und Einsichten. - Bern: h.e.p Verlag. 
Dirksmeier, P. (2007): Der husserlsche Bildbegriff als theoretische Grundlage der reflexiven Fotografie: Ein Beitrag zur visuellen Methodologie in der Humangeografie. - Social Geography 2, 1:1-10.

Erne, F., Ghilardi, V., Rüttimann, K. \& B. Steinmann (2003): Medienresonanz des Biosphärenreservates Entlebuch. Analyse der Medienberichtersattung und des Medienimages des BRE. - Projektarbeit Studiengang Wirtschaftskommunikation, Luzern: Hochschule für Wirtschaft.

Flitner, M. (1999): Im Bilderwald - politische Ökologie und die Ordnungen des Blicks. - In: Zeitschrift für Wirtschaftsgeographie 43, 3-4: 169-183.

Gast-Gampe, M. (1993): Einstellungen, Stereotype, Vorurteile. - In: HAHN, H. \& H.J. KagelmanN (Hrsg.): Tourismuspsychologie und Tourismussoziologie: Ein Handbuch zur Tourismuswissenschaft. - München: Quintessenz Verlag: 127-131.

Giddens, A. (1984): The constitution of society. Outline of the Theory of Structuration. - Cambridge: Polity Press.

Hard, G. (1994): Regionalisierung. - In: Wentz, M. (Hrsg.): Region. - Frankfurt am Main, New York: Campus-Verlag: 53-57.

Hennig, C. (1999): Reiselust - Touristen, Tourismus und Urlaubskultur. - Frankfurt am Main: Insel Verlag. Luhmann, N. (1996): Die Realität der Massenmedien. - Opladen: Westdeutscher Verlag.

MaAr, C. \& H. Burda (Hrsg.) (2004): Iconic Turn - Die neue Macht der Bilder. - Köln: DuMont.

Mitchell, W.J.T. (1990/Orig. 1986): Was ist ein Bild? - In: BoHn, V. (Hrsg.): Bildlichkeit. - Frankfurt am Main: Suhrkamp: 17-68.

Müller, U. (2007): Die Kraft der Bilder in der nachhaltigen Entwicklung. Die Fallbeispiele UNESCO Biosphäre Entlebuch und UNESCO Weltnaturerbe Jungfrau-Aletsch-Bietschhorn. - Zürich: vdf Hochschulverlag AG.

Müller, U. \& N. BACKhaus (2007): The Entlebuchers: people from the back of beyond? - In: Social Geography 2, 1: 11-28.

Probst, L. (1999): «Irgendwie heimelig» und «manchmal ziemlich langweilig». - In: Scagnet, E., Duss-STuder, H., Ehrler, J., Küng, J., Lohri, P., Probst, L. \& A. SchmidiGER (Hrsg.): Lebenszeiten im Entlebuch: das Amt Entlebuch. - Schüpfheim: Verlag Druckerei Schüpfheim: o.S.

ReIche, J. (2003): Macht der Bilder. - In: HAus DER Geschichte DER BundesRepublik Deutschland (Hrsg.): X für U - Bilder, die lügen. - Bonn: Bundeszentrale für Politische Bildung: 10-19.

Rose, G. (2001): Visual methodologies - An introduction to the interpretation of visual materials. - London: Sage.

Schmid, A., Ruoss, E. \& H. ElsAsser (2004): UNESCO Biosphäre Entlebuch: Modell für eine nachhaltige Regionalentwicklung? - Konzept Zielerreichungskontrolle. - In: Geographica Helvetica 59, 2: 144-153.
Scholz, O.R. (2004): Bild, Darstellung, Zeichen: Philosophische Theorien bildlicher Darstellung. - Frankfurt am Main: Klostermann.

Sontag, S. (1978): Über Fotografie. - München, Wien: Carl Hanser.

StÄmpfli, R. (2007): Die Macht der Bilder: Wer die Bilder beherrscht, beherrscht die Köpfe. - In: KMUMagazin 1, Februar 2007: 98-91.

Stremlow, M. (1998): Die Alpen aus der Untersicht: Von der Verheissung der nahen Fremde zur Sportarena. Kontinuität und Wandel von Alpenbildern seit 1700. - Bern: Haupt Verlag.

UNESCO (1995): The Seville strategy for biosphere reserves. - http://www.unesco.org/mab/BRs/pdf/Strategy.pdf 10.10.2006.

URRY, J. (2002): The tourist gaze. - London: Sage.

WemF AG (2003): MACH Basic. - http://www.wemf. $\mathrm{ch} / \mathrm{d} /$ studien/machbasic.shtml 10.4.2004.

Werlen, B. (2003): Cultural turn in den Humanwissenschaften und Geographie. - In: Berichte zur deutschen Landeskunde 77, 1:35-52.

Wicki, O. \& A. Kaufmann (2003): Aus alter Zeit: Geschichten und Bilder aus dem Entlebuch. - Schüpfheim: Druckerei Schüpfheim AG.

WIESING, L. (2005): Artifizielle Präsenz: Studien zur Philosophie des Bildes. - Frankfurt am Main: Suhrkamp.

\section{Zusammenfassung: Die UNESCO Biosphäre Entlebuch im Bild. Präsentation einer Methode zur Analyse visueller Regionalisierungen}

Im heutigen visuell dominierten Zeitalter prägen und formen visuelle (Re-)Präsentationen die Wahrnehmung und Aneignung von Regionen massgeblich. Bilder vermitteln Vorstellungen davon, welche Beziehungen $\mathrm{zu}$ einem bestimmten Raum eingenommen werden können und sollen. Sie zeigen, was sich in den Regionen tun lässt, welche Raumnutzungen vorhanden, möglich und erwünscht sind. In diesem Artikel wird eine Methode vorgestellt, durch die die potenzielle Raumwirksamkeit visueller Bilder erfasst werden kann. Sie ermöglicht, bildreiche Präsentationen von «Räumen» in ein übersichtliches Gesamtbild zu übersetzen und so unterschiedliche, unter Umständen in Konflikt zueinander stehende Raumaneignungsweisen herauszuarbeiten. Demonstriert wird die Methode anhand unterschiedlicher Publikationen über die UNESCO Biosphäre Entlebuch. Die Analyse offenbart die Differenz zwischen alltagsnaher Innensicht und romantisierender Aussensicht und dokumentiert einen im Ansatz unkonventionellen, Klischees aufbrechenden touristischen Auftritt.

\section{Abstract: The Entlebuch UNESCO Biosphere Reserve in pictures. Presentation of a method of analysing visual regionalisations}

In today's image-dominated age, visual (re)presentations have a special formative power over the way 
regions are perceived and appropriated. Images convey ideas about the relations people can and should develop with a particular space. They show what can be done in these regions and which uses of space already exist, as well as those that might be possible or desirable. In this article, a method of capturing the potential spatial effect of visual images is proposed. This method makes it possible to obtain a clear overview of presentations of space that contain a large amount of visual imagery and thus bring out various means of appropriating the space that might possibly conflict with each other. This method is applied to various publications about the Entlebuch UNESCO Biosphere. Analysis of the results reveals the difference between the everyday perception of insiders and the more romantic view of those from outside, as well as recording an unconventional approach to presenting the area to tourists that defies clichés.

\section{Résumé: La réserve de biosphère Entlebuch (UNESCO) en images. Présentation d'une méthode d'analyse visuelle régionale}

Dans notre époque dominée par les images, les représentations visuelles jouent un rôle déterminant dans notre façon de percevoir et de s'approprier les régions. Les images véhiculent des notions relatives aux relations que l'on peut ou que l'on doit avoir avec un espace donné. Elles montrent ce qu'il est possible de faire dans une région, quelles utilisations de l'espace existent déjà, ainsi que celles qui sont possibles ou souhaitables. Dans cet article, une méthode permettant de comprendre les effets potentiels de certaines images sur un espace est présentée. Elle permet également d'obtenir une vue d'ensemble claire de certains espaces iconographiquement très chargés et de mettre en relief les modes d'appropriation de l'espace qui peuvent parfois entrer en conflit les uns avec les autres. La méthode est illustrée à l'aide d'un certain nombre de publications présentant la réserve de biosphère d'Entlebuch mise en œuvre par l'UNESCO. Cette analyse révèle la différence entre une vue quotidienne intérieure et une vue romantique venant de l'extérieur et montre l'utilité d'une approche touristique non conventionnelle brisant les clichés.

Dr. Urs Müller, PD Dr. Norman Backhaus, Geographisches Institut, Abteilung Humangeographie, Prof. Dr. Hans Elsasser, Geographisches Institut, Abteilung Wirtschaftsgeographie, Prof. Dr. Ulrike Müller-Böker, Geographisches Institut, Abteilung Humangeographie, Universität Zürich, Winterthurerstrasse 190, CH8057 Zürich, Schweiz.

e-mail:

urs.mueller@geo.uzh.ch norman.backhaus@geo.uhz.ch hans.elsasser@geo.uzh.ch ulrike.mueller-boeker@geo.uzh.ch

Manuskripteingang/received/manuscrit entré le 5.3.2007

Annahme zum Druck/accepted for publication/accepté pour l'impression: 30.11 .2007 